\title{
Living longer, staying healthy
}

Lifespan in nematodes is markedly extended when an unusual combination of aging components is engineered into their genome.

In the wild, nematode life often ends in a predator's jaws. Even evading those encounters, Caenorhabditis elegans lives just a fast and furious 2 weeks. Engineered worms in Stuart Kim's lab at Stanford University Medical Center, however, sail past their 1-month birthday, reaching $230 \%$ of their natural lifespan.

When postdoctoral fellow Dror Sagi approached Kim with the "wild and crazy" idea to not just extend an organism's life but engineer healthy aging, Kim says he found it conceptually surprising and courageous. For example, researchers studying aging had not previously added zebrafish genes to the nematode genome, a step that seemed more likely to cut the worm's life short than extend it. This work - 5 years in the making-shows the opposite to be true.

Applying a stepwise genome engineering approach to study how aging can be influenced, the researchers overexpressed specific nematode and zebrafish genes. Each engineered module endowed the animals with longer life. In human terms, the babies and children developed normally and "turned 18 on time," but then came a shift, says Kim. "We're keeping worms in middle age for a long time," he says, adding that this result is a promising avenue for future research.

With their successive experiments, the scientists added individual components into the nematode genome: worm genes with known life-extension qualities, genes in anti-aging pathways, non-native genes with orthologs in the nematode, and a gene from zebrafish with a function not found in the worm. Achieving gene expression across species boundaries is trivial, says Sagi, a physicist drawn to bioengineering and aging research. Less trivial is creating a modular, anti-aging gene expression strategy. Although the team considered human genes, they chose zebrafish for physiological reasons: both fish and worms are cold-blooded, and they live in a similar temperature range, says Sagi.

The team created expression vectors for nematode genes that are known to be life extending when overexpressed: $h s f-1$, aakg-2 and sod-1. With a view to different

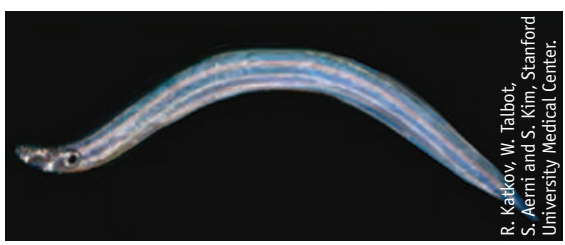

A fictitious animal, a fishworm, is a symbol of experimental success for the authors.

functionality, they reached for pathway players such as $\operatorname{lm} p$-2, known to be part of cellular protein cleanup. They also tested overexpressed vertebrate genes: the zebrafish genes sod1, which encodes a free-radical destroyer, and $u c p 2$, which lacks a worm ortholog.

The gene $u c p 2$ encodes an uncoupling protein that appears to slow aging through mitochondrial action and, in warm-blooded mammals, helps manage thermoregulation. The researchers also picked the zebrafish lyz gene, an element of vertebrate-class defense weaponry lacking in C. elegans. The gene encodes an enzyme that thwarts pathogenic bacteria, which also plague nematodes.

The researchers found that worm lifespan increased by about one-third with single components, and combinations ratcheted up the effect. With two components, the nematodes lived around 70\% longer; triple elements led to a $95 \%$ gain; and the quadruple constellation delivered a $130 \%$ lifespan boost.

To obtain evidence of physiological changes in the transgenic animals, the team set up several types of assays. Success turns the assays into the experimental bottleneck because they can take as long as 3 months, the scientists say. As the worms live longer, plates get crowded, and the risk of contamination soars.

The researchers believe that their work opens the door to engineering genomes that include complex functions and pathways derived from other species. "Maybe we can just install them as a module into a worm and can expect them to work out of context and to benefit the worm," Kim says. Although a bottled fountain of youth for vertebrates is not yet reality, this genome infusion offers a sip of future possibilities.

\section{Vivien Marx}

\section{RESEARCH PAPERS}

Sagi, D. \& Kim, S.K. An engineering approach to extending lifespan in C. elegans. PLoS Genet. 8 e1002780 (2012). 